\title{
An investigation of the topology and structure of constant- $\alpha$ force-free fields
}

\author{
G. J. D. Petrie ${ }^{1, \star}$ and R. M. Lothian ${ }^{2}$ \\ 1 FORTH and Department of Physics, University of Crete, PO Box 2208, 71003 Heraklion, Crete, Greece \\ e-mail: gordonp@physics.uoc.gr \\ 2 School of Computing, The Robert Gordon University, St. Andrews Street, Aberdeen AB25 1HG, UK \\ e-mail: rml@scms.rgu.ac.uk
}

Received 27 July 2001 / Accepted 7 October 2002

\begin{abstract}
Coronal field structures corresponding to specified normal fields on the photosphere are investigated using a forcefree model. The force-free parameter is insufficient to determine the field uniquely, and so a second parameter representing the contribution of the complementary Green's function is included. For isolated loops represented by a single source-sink pair, the influence of the two parameters on field structure is comparable. Effects on the topology (as measured by field line connectivity) of multiple source configurations are also found to be comparable. It is notable that some configurations are more stable than others to variation of either parameter. The results of this study are compared with previous magnetic charge topology studies involving only the force-free parameter.
\end{abstract}

Key words. Sun: Corona - Sun: magnetic fields - methods : analytical

\section{Introduction}

The highly structured nature of the solar corona is largely due to the presence there of strong magnetic fields. Unfortunately, it is extremely difficult to measure magnetic fields in the rarefied coronal plasma. However, it is relatively easy to measure the line-of-sight component of the photospheric field. Consequently, there has been considerable interest in methods of extrapolating the photospheric field into the corona. The solution of this type of boundary value problem is also of interest from a modelling perspective. In order to make progress with this problem, a number of simplifying assumptions are necessary. It is normal to use the low plasma pressure and large scale height in the corona as a justification for treating the magnetic field as force-free. This requires that currents are field-aligned, obeying the equation:

$\mathbf{J}=\alpha \mathbf{B}$,

where $\mathbf{J}$ is the current density, $\mathbf{B}$ is the magnetic field and the force-free parameter $\alpha$ is a function of position, but is constant along a given field line. Since plasma velocities are nonrelativistic, we may ignore the displacement current and express Eq. (1) as

$\nabla \times \mathbf{B}=\alpha \mathbf{B}$.

Send offprint requests to: G. J. D. Petrie, e-mail: gordonp@physics.uoc.gr

* Present address: IASA and Section of Astrophysics, Astronomy and Mechanics, Department of Physics, University of Athens, Panepistimiopolis, 15784 Zografos, Athens, Greece.
In general, such non-linear force-free fields are difficult to calculate and there is a lack of theorems concerning the existence and uniqueness of solutions. Consequently, two simplified problems have been commonly considered. Schmidt (1964) solved for the potential $(\alpha=0)$ field, effectively declaring coronal currents to be negligible. This method has the advantage that there is a unique potential field corresponding to a given photospheric normal field distribution. It is less restrictive to assume that $\alpha$ is a non-zero constant. While the global solar field is not constant- $\alpha$, it is a useful local approximation and the freedom of choice of $\alpha$ gives some flexibility in modelling the field. Furthermore, Taylor's (1974) theory tells us that a non-linear field subjected to small scale dissipation will relax to a constant- $\alpha$ state. With $\alpha$ constant, we may solve the Helmholtz equation

$\left(\nabla^{2}+\alpha^{2}\right) \mathbf{B}=\mathbf{0}$.

However, there is no unique solution corresponding to a given photospheric normal field and value of $\alpha$. Instead, we may include arbitrary amounts of the complementary solution, which satisfies (3) and homogeneous boundary conditions. In this work, we employ a Green's function approach, but it should be noted that the non-uniqueness is a property of the boundary value problem, not an artefact of the solution method. Alissandrakis (1981) briefly discusses the manner in which the non-uniqueness manifests itself in the fast Fourier transform (FFT) solution. Gary (1989) compares the Green's function and FFT methods. The Green's function solution, including the non-unique complementary part, was first given by 
Chiu \& Hilton (1977). We follow those authors in treating the photosphere as an infinite plane. In practice, the solution is only useful over lengths smaller than the solar radius and no greater than $1 / \alpha$. (It is, however, worth noting that global solutions can be constructed by pasting many such local solutions together, Clegg et al. 1999.) Semel (1988) generalised the Green's function solution from a specified normal field to an oblique field, allowing it to be applied to the observed line-of-sight field.

A number of authors have suggested ways to select a unique solution. Barbosa (1977) specified zero field at a given height above the photosphere. However, there is no good physical reason to do this and the choice of height remains arbitrary. In Chiu \& Hilton's original paper, they showed that a unique solution could be obtained by specifying the observable $\tan \psi=\frac{B_{y}}{B_{x}}$. However, since this solution is in the form of an integro-differential equation, it has never actually been applied. Furthermore, there are practical difficulties associated with this approach. The transverse components cannot be measured as accurately as the line-of-sight component. In any case, neither the measured nor the real field are exactly force-free, let alone linear. To deal with these difficulties, variational schemes have been devised by Amari et al. (1997) and Wheatland (1999). In particular, the latter develops his method within the Green's function formalism. The line-of-sight component is matched to that observed and the remaining freedom in the solution is used to minimise the deviation between the observed and modelled transverse components. This method provides a practical solution to the reconstruction problem when all three components have been measured. However, it is often the case that only one component is available. In practice, it has been common to ignore the complementary function. This has more to do with convenience than any physical justification.

A related issue is the use of constant- $\alpha$ fields in magnetic charge topology (the phrase is Longcope's 1996) studies. Lothian \& Browning (1995) studied a symmetrical configuration of two sources and two sinks. In the potential field solution, the sources and sinks were connected in pairs, with flux from any source going to only one sink. Introduction of a finite $\alpha$ broke this symmetry and allowed flux to transfer from one sink to another. In their model, Lothian \& Browning (1995) interpreted this as reconnection occurring during an implicit relaxation process. In a recent treatment of a similar configuration, Hudson \& Wheatland (1999) emphasised the topological difference between the potential and constant- $\alpha$ fields. The plane $x=0$ is a separatrix surface in the potential case, but this surface disappears at non-zero $\alpha$. These authors suggested that such changes in topology could be seen as a weakness of the constant- $\alpha$ model. In response, Brown $\&$ Priest (2000) pointed out that the topological instability of such configurations is due to their artificially high symmetry. However, all of these authors assumed that there was no contribution from the complementary solution.

In this paper, we investigate the effect that including a contribution from the complementary function has on some simple magnetic configurations. For convenience, we restrict the form of this contribution, as described in Sect. 2, in such a way that it can be specified by a single parameter. In Sect. 3, we first examine loop structures represented by simple source-sink pairs. Then we move on to four-source configurations, investigating the effect on field line connectivity of including some complementary solution. In the light of the previous work mentioned above, we study two configurations, one being more topologically stable than the other. In Sect. 4, we draw some conclusions about the consequences of the common neglect of the complementary solution.

\section{Mathematical development}

In adopting Cartesian coordinates we assume that the structures described are small compared to the radius of the sun. In this approximation we roughly identify the surface $z=0$ with the photosphere, the base of the solar atmosphere. Given a profile for the vertical component of the magnetic field $B_{z}$ on the photosphere $z=0$, either from a magnetogram or fixed in advanced by the theorist, we seek to extrapolate this field into the corona $z>0$. This problem has been considered by many researchers including Chiu \& Hilton (1977). They developed an eigenfunction expansion from which they were able to obtain a general solution in Green's function form

$$
\begin{aligned}
\mathbf{B}(x, y, z)= & \frac{1}{2 \pi} \int_{z^{\prime}=0} \mathbf{G}\left(x-x^{\prime}, y-y^{\prime}, z\right) B_{z}\left(x^{\prime}, y^{\prime}, 0\right) \mathrm{d} x^{\prime} \mathrm{d} y^{\prime} \\
& +\frac{1}{2 \pi} \int_{z^{\prime}=0} \tilde{\mathbf{G}}\left(x-x^{\prime}, y-y^{\prime}, z\right) C\left(x^{\prime}, y^{\prime}\right) \mathrm{d} x^{\prime} \mathrm{d} y^{\prime}
\end{aligned}
$$

where $\mathbf{G}=\left(G_{x}, G_{y}, G_{z}\right)$ and $\tilde{\mathbf{G}}=\left(\tilde{G}_{x}, \tilde{G}_{y}, \tilde{G}_{z}\right)$ and $C(x, y)$ is an arbitrary function of the source coordinates. Here dashed coordinates refer to source points and undashed coordinates are the spatial coordinates of the magnetic field. The function $\mathbf{G}$ is the familiar Green's function (source solution) and $\tilde{\mathbf{G}}$ is the “complementary" Green's function necessary to make Eq. (4) a general solution for the magnetic field. For any given value for $\alpha$, boundary data for $B_{z}$ fix the part of the field corresponding to $\mathbf{G}$, but the complementary Green's function $\tilde{\mathbf{G}}$ is a homogeneous solution whose contribution to the field is not determined by the boundary conditions, except for the special case $\alpha=0$, and the problem as it stands is ill-posed. Wheatland (1999)'s Eq. (12) shows how the arbitrary function $C(x, y)$ is related to the tangential field components on the boundary and, as discussed in Sect. 1, this and other attempts have been made to eliminate the difficulty of ill-posedness by including these or other additional boundary conditions. However, it remains the case that the most easily and accurately obtained observational data correspond to the ill-posed problem. It has been common to follow Chiu \& Hilton (1977) in simply setting $C(x, y)$ equal to zero everywhere. Here, we investigate the effect of including some contribution from the complementary function. The arbitrary function $C(x, y)$ represents a wide range of possibilities for this contribution. In order to make our study practicable, we shall restrict our attention to a particular form for this function, explicitly

$C(x, y)=b B_{z}(x, y)$,

where $b$ is a real parameter. The case $b=0$ imposes the condition $\frac{\partial B_{z}}{\partial z}=0$ everywhere on the boundary, except at sources 
of $\mathbf{B}$, where it is fixed by the strength of the source. Our generalisation allows variation of the value of $\frac{\partial B_{z}}{\partial z}$ at source points. Hence, the behaviour of the field near its sources can be altered. This allows us to study the effect on the field structure of changes in $\alpha$ and $b$. Using the sources of the normal field as sources of the additional complementary field seems appropriate in the context of the MCT approach. Of course, there are many other possible forms for $C(x, y)$, but our choice should indicate the significance of the complementary function.

The Green's function components $G_{x}, G_{y}$ and $G_{z}$ are of the form (see Chiu \& Hilton 1977)

$$
\begin{aligned}
G_{x} & =\frac{\partial R}{\partial x} \frac{\partial \bar{\Gamma}}{\partial z}+\alpha \frac{\partial R}{\partial y} \bar{\Gamma}, \\
G_{y} & =\frac{\partial R}{\partial y} \frac{\partial \bar{\Gamma}}{\partial z}-\alpha \frac{\partial R}{\partial x} \bar{\Gamma}, \\
G_{z} & =-\frac{\partial \bar{\Gamma}}{\partial R}-\frac{\bar{\Gamma}}{R},
\end{aligned}
$$

where

$\bar{\Gamma}=\frac{z}{R r} \cos (\alpha r)-\frac{1}{R} \cos (\alpha z)$,

with $r^{2}=x^{2}+y^{2}+z^{2}$. Meanwhile $\tilde{\mathbf{G}}$ has the same structure Eqs. (7-8) as $\mathbf{G}$ with $\bar{\Gamma}$ replaced by a function $\tilde{\Gamma}$ whose expression is

$\tilde{\Gamma}=\frac{z}{R r} \sin (\alpha r)-\frac{1}{R} \sin (\alpha z)$

We make the popular simplifying assumption that the magnetic flux is generated by a finite sum of point sources on the photosphere $z=0$ so that

$B_{z}(x, y, 0)=\sum_{i} w_{i} \delta\left(x-x_{i}, y-y_{i}\right)$,

where $w_{i}$ are the weights of the sources. With this assumption the integral expression for $\mathbf{B}$ simplifies to the finite sum

$\mathbf{B}(x, y, z)=\sum_{i} \mathbf{G}\left(x-x_{i}, y-y_{i}, z\right)$

This assumption is adopted for simplicity by Chiu \& Hilton (1977) and others despite the consequent singularities on the photosphere. These singularities lie in the region of interest, $z \geq 0$, and therefore cannot be ignored. Two possible ways round this are to use instead of point sources discrete sources of finite flux (see Lothian \& Browning 1995) or to use point sources positioned a small distance below the photosphere (see Petrie \& Neukirch 2000) and carefully weight them so that the desired profile at $z=0$ is achieved (see Petrie 2000, where this method is used extensively in the fitting of a related class of non-force-free equilibria to magnetogram data). However, since surface point-source solutions can give a good idea of the global connectivity of a system and are very simple to apply we adopt this method in the current project.

\section{The effect of the complementary Green's function on field Line connectivity}

Figures 1-3 indicate how the complementary Green's function is capable of affecting the properties of two simple systems. For each example configuration selected force-free magnetic field lines are plotted for zero and non-zero $b$.

We show one example with two sources and one example with four sources. In the two-source example, a unit point magnetic flux source is placed at $(x, y, z)=(-1.0,1.0,0.0)$ and a unit point sink at $(x, y, z)=(1.0,-1.0,0.0)$. Figure 1 shows the cases $\alpha=1.0, b=0.0$ (top pictures) and $\alpha=0.2, b=-3.0$ (bottom pictures). In each case eight field lines are plotted with start points arranged in a circle on $z=0$ of radius 0.2 units centred at the source, the same set of start points being used in each case. Comparing the two cases, the effect of $b$ is clearly visible. Where $\alpha$ tends to give the magnetic field some visible twist and curvature, in the case with negative $b$ the field has a reversed visible twist and curvature and the field lines are lower than in the case with zero $b$. Cases with various values of $b$, positive and negative, and various values of $\alpha$ have been explored. A general pattern emerges. When $b$ has the same sign as $\alpha$, the field tends to acquire additional twist. Conversely, when $b$ and $\alpha$ have opposite signs, the twist decreases as $b$ is increased and can eventually be reversed.

In the four-source example, point unit flux sources are placed at $(x, y, z)=(-1.0,1.0,0.0)$ and $(1.0,1.0,0.0)$, and unit point flux sinks at $(x, y, z)=(-1.0,-1.0,0.0)$ and $(1.0,-1.0,0.0)$. Figure 2 shows the case $\alpha=1.0, b=0.0$ and Fig. 3 shows the case $\alpha=1.0, b=-10.0$. Again, making $b$ non-zero has a visible effect on the magnetic field. In both figures eight field lines are plotted beginning near the left source $(x, y, z)=(-1.0,1.0,0.0)$ and a further eight are plotted with start points near the right source $(x, y, z)=(1.0,1.0,0.0)$ (the start points are arranged in two circles on $z=0$ of radius 0.2 units, one circle centred on each source). In Fig. 2 the influence of $\alpha$ is evident in that all of the displayed field lines leaving the neighbourhood of the left source reach the neighbourhood of the right sink at $(x, y, z)=(1.0,-1.0,0.0)$, while all lines from close to the right source reach close to the left sink at $(x, y, z)=(-1.0,-1.0,0.0)$. This is in contrast to the potential case where all field lines leaving the left source end at the left sink and all leaving the right source reach the right sink (see Lothian \& Browning 1995; Hudson \& Wheatland 1999; Brown \& Priest 2000). In Fig. 3, where the same start points are used as in Fig. 2, the non-zero value of $b$ clearly acts against this effect of $\alpha$ and all field lines displayed leaving the neighbourhood of the left source reach the neighbourhood of the left sink and all shown from the neighbourhood of the right source reach the neighbourhood of the right sink.

From the field lines shown in Figs. 1-3, it is evident that varying the contribution of the complementary Green's function $\tilde{\mathbf{G}}$ to the magnetic field can significantly change the appearance of the field. In order to explore the effect of $\tilde{\mathbf{G}}$ in more quantitative terms we take two simple four-source configurations and calculate the effect of varying $\tilde{\mathbf{G}}$ on each configuration. The two chosen configurations are the following: one where two unit point flux sources and two unit point sinks are 

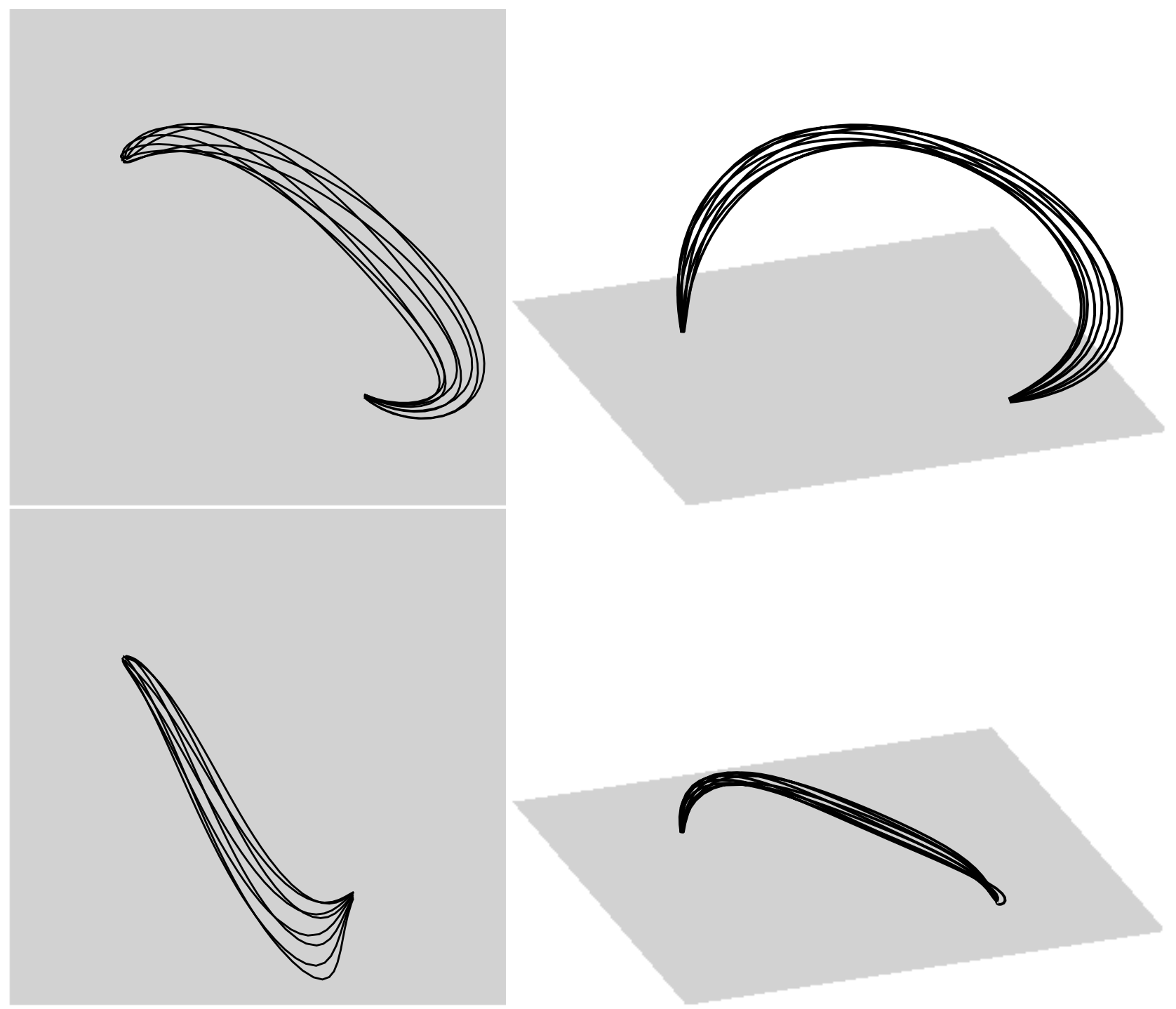

Fig. 1. The effect of the complementary Green's function on a two-source system. A unit point magnetic flux source is placed at $(x, y, z)=$ $(-1.0,1.0,0.0)$ and a unit point sink is placed at $(x, y, z)=(1.0,-1.0,0.0)$. The top pictures show the case $\alpha=1.0, b=0.0$ and the bottom pictures show the case $\alpha=1.0, b=-3$.0. In each case eight field lines are plotted from start points on a circle with centre $(x, y, z)=$ $(-1.0,1.0,0.1)$ and radius 0.2 . The influence of the complementary Green's function is evident in the different curvature and twist of the field lines in the bottom pictures compared to those in the top pictures. Note also that the lines are lower in the bottom pictures than in the top pictures.

arranged on $z=0$ in a square with the sources at the top vertices and the sinks at the bottom vertices, and a second configuration where two unit point sources and two unit point sinks are arranged alternately around the vertices of a square on $z=0$. The first configuration is similar to those studied by previous authors. More precisely, the same-side configuration has unit point flux sources at $P_{1}=(-1,1,0)$ and $P_{2}=(1,1,0)$ and unit point flux sinks at $P_{3}=(-1,-1,0)$ and $P_{4}=(1,-1,0)$ while the alternating configuration has unit point flux sources at $P_{1}=(-1,1,0)$ and $P_{4}=(1,-1,0)$ and unit point flux sinks at $P_{2}=(1,1,0)$ and $P_{3}=(-1,-1,0)$. For each configuration a measure of the field line connectivity is obtained by starting a large number of field lines near $P_{1}$, following each line until it arrives to within a very small distance 0.01 of one of the two flux sinks in the configuration, and recording how the field lines divide themselves between the two sinks for different contributions from $\tilde{\mathbf{G}}$ and different values of $\alpha$.

In order to make these measurements as representative as possible we distribute the field line start points around $P_{1}$ as evenly as we can, although it is not possible to anticipate the distribution of the end points and to arrange this also to be uniform. This even distribution of the field line start points over a very small hemisphere of radius 0.01 centred at the flux source is chosen on assuming that, the hemisphere being so close to the source, the field strength across it is very nearly uniform. Records of field strengths at start points indicate that this is true. Thus we can assume that this distribution of field lines represents to a good approximation the distribution of flux emerging from the source. This approach to approximating the flux output from the source is the same as that used in 

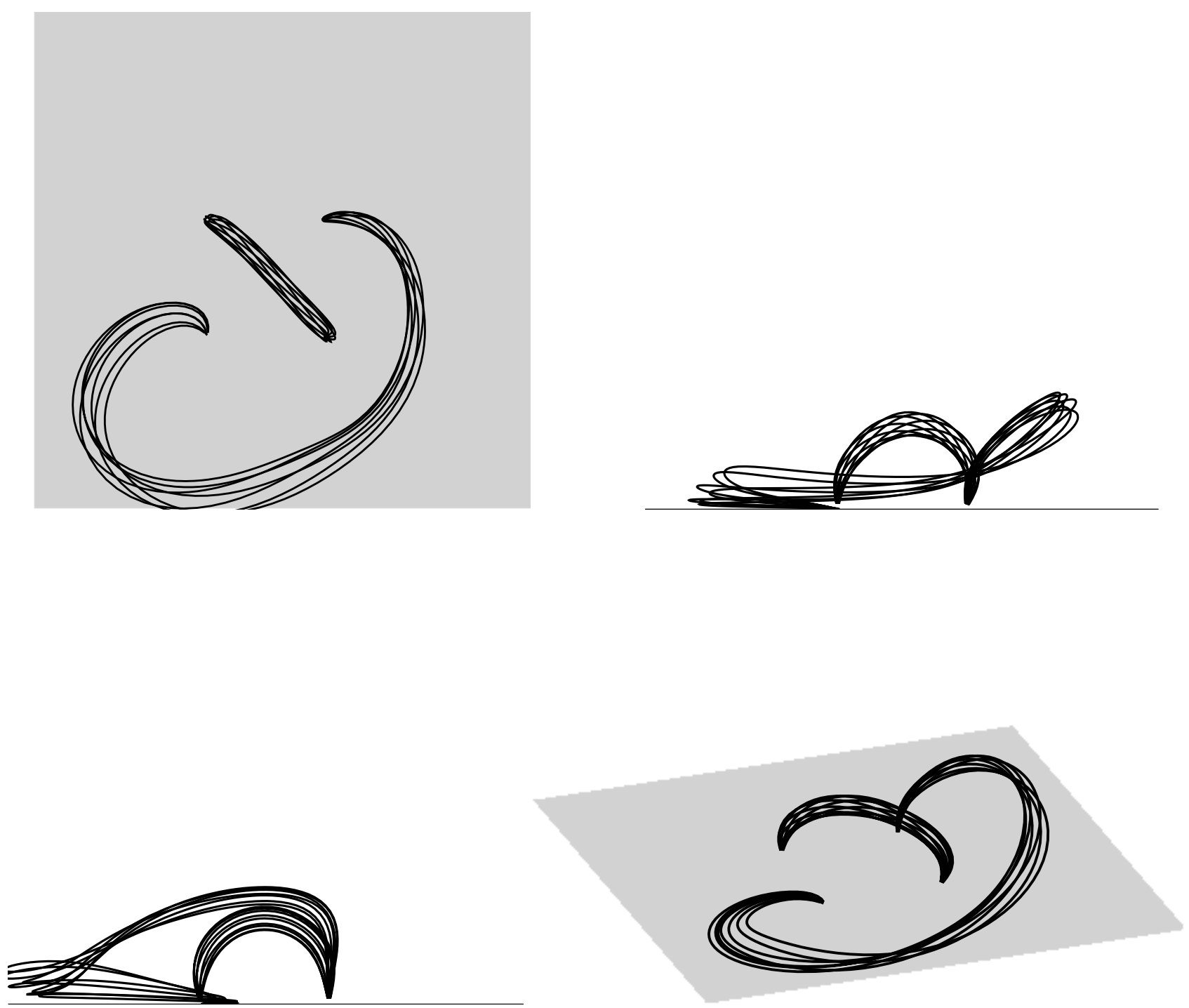

Fig. 2. Four-source example with $\alpha=1.0$ and $b=0.0$ viewed from above (top left picture), along $y$-axis (top right), along $x$-axis (bottom left) and an oblique view (bottom right). The effect of the complementary Green's function on the four-source system can be seen by comparing this figure with Fig. 3. Unit point magnetic flux sources are put at $(x, y, z)=(-1,1,0),(1,1,0)$ and unit point flux sinks at $(x, y, z)=(-1,-1,0)$, $(1,-1,0)$. At each source eight field lines are plotted from start points on a circle with centre the source and radius 0.2 . In this figure, all displayed field lines from close to the left source terminate close to the right sink and those from close to the right source terminate close to the left sink, whereas all from near the left source terminate close to the left sink and all from near the right source terminate near the right sink in the $b=-10.0$ case of Fig. 3 . This suggests that the change in the value of $b$ results in a significant flux transfer between the two sinks.

Hudson \& Wheatland (1999)'s Sect. 3.3.2 except that Hudson $\&$ Wheatland take as start points five hundred random points inside their small hemisphere of radius 0.01 centred at the source.

The connectivity graphs for both same-side and alternate configurations are shown in Fig. 4. In the same-side configuration, varying $\alpha$ has a clear effect on the field line connectivity. With a potential field, all field lines from the left source arrive at the left sink. For positive $\alpha$, field lines tend to spiral away from a source in an anti-clockwise direction, and this causes some of the flux to switch from the left sink to the right sink. The amount of flux switching increases with $\alpha$ until, at about $\alpha=0.5$, just as much flux from the left source reaches the right sink as reaches the left sink. For $\alpha>0.5$ more flux from the left source arrives at the right sink than at the left sink. The right half of Fig. 4 (left) is in agreement with Hudson \& Wheatland (1999)'s Fig. 3, where they quantitatively demonstrated the association between the variation of $\alpha$ and flux transfer between sinks.

For negative $\alpha$, exactly the same is true with regard to flux from the right source switching from the right sink to the left sink, so that any flux switching from the left sink to the right sink has to travel a long way. Some of the flux could not be traced to either sink. In previous work, such lost flux has been attributed to finite integration times. The assumption, implicit or explicit, has been that all flux from the sources must connect them to the sinks. In that case, the true version of 

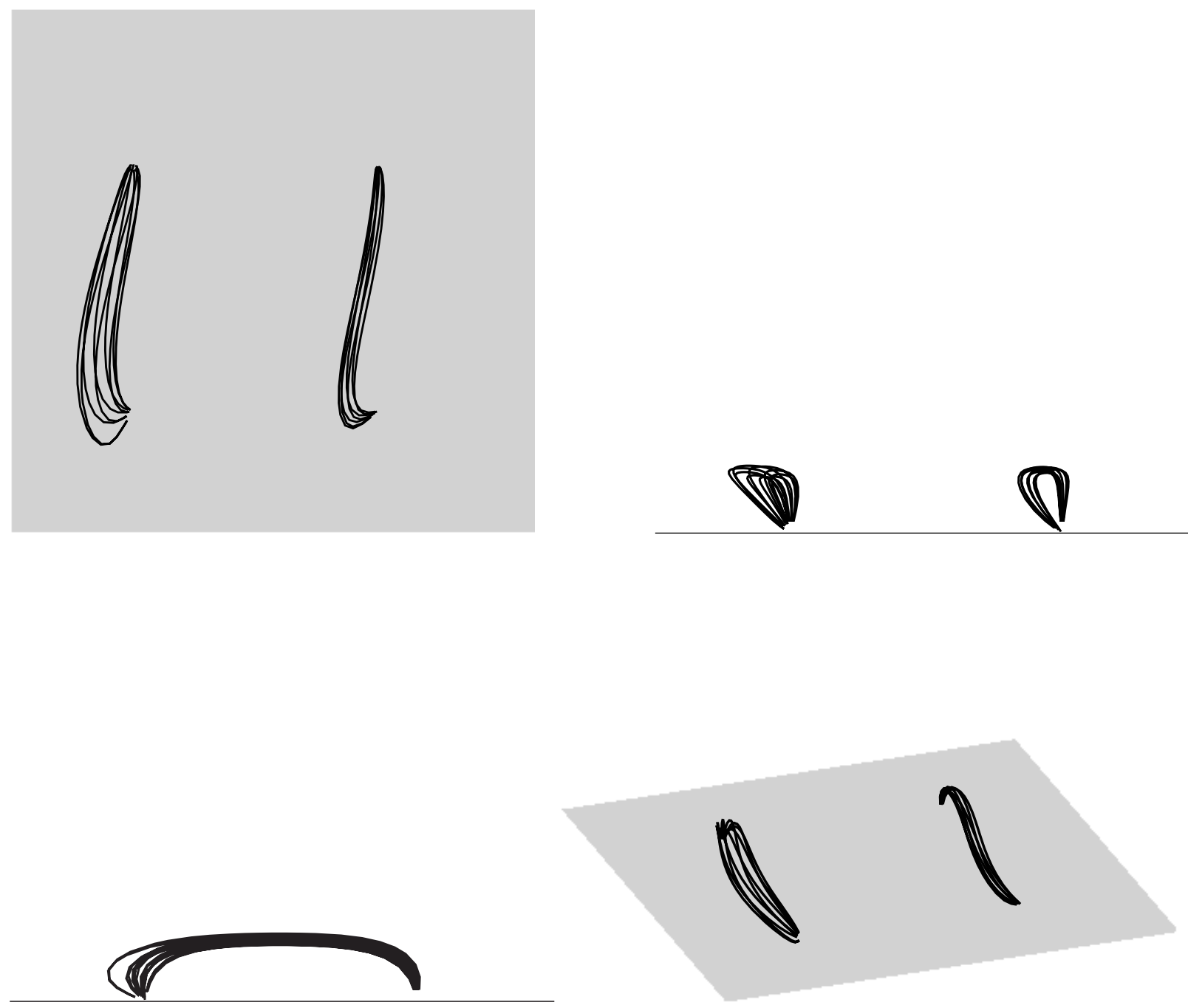

Fig. 3. Four-source example with $\alpha=1.0$ and $b=-10.0$ viewed from above (top left picture), along $y$-axis (top right), along $x$-axis (bottom left) and an oblique view (bottom right). The effect of the complementary Green's function on a four-source system can be seen by comparing this figure with Fig. 2. Unit point magnetic flux sources are put at $(x, y, z)=(-1,1,0),(1,1,0)$ and unit point flux sinks at $(x, y, z)=(-1,-1,0)$, $(1,-1,0)$. At each source eight field lines are plotted from start points on a circle with centre the source and radius 0.2 . In the figure, all displayed field lines from close to the left source terminate close to the left sink and those from close to the right source terminate near the right sink, unlike in the $b=0$ case of Fig. 2, suggesting that the change in the value of $b$ results in a significant flux transfer between the two sinks. Here, as in Fig. 1, the non-zero $b$ case's field lines appear to be lower than those of the $b=0$ case.

Fig. 4 (left) would be completely symmetrical. However, the authors are unaware of any proof that this is true. In fact, this lost flux does not conflict with known results and our asymmetric graph of connectivity with $\alpha$ (Fig. 4, left) can be seen to correspond with the asymmetric non-potential separatrices of Brown \& Priest (2000)'s Fig. 2, where the top left source and bottom right sink are inside the finite connectivity region while the top right source and bottom left sink are outside are outside this finite region or on the separatrix. This would suggest e.g. that different connectivity properties are possible for field lines from the top left source to the bottom left sink than for lines from the top left source to the bottom right sink as we show in our Fig. 4 (left). Examining Brown \& Priest (2000)'s Fig. 2 in more detail, their bottom pictures shows the case $\alpha=+0.5$ where the top left source lies within one of the finite connectivity lobes, and it can be seen in the three-dimensional topology plot on the right that lines from the top left source can travel to both bottom left and bottom right sinks without travelling far. The $\alpha=-0.5$ case would be similar except that the finite connectivity lobes would change their top left-bottom right orientation to a top right-bottom left orientation so that now the top left source would lie outside the finite connectivity region as in Brown \& Priest (2000)'s Fig. 5. Lines from the top left source would have to travel a long way to the bottom right sink here, as lines in Brown \& Priest (2000)'s Fig. 2 from the top right source to the bottom left sink must do. In this way Brown \& Priest (2000) seem to support us at least as far as saying that the situation is not symmetrical in $\alpha$ through clearly distinguishing finite and infinite connectivity regions. Even though no proof that the flux travels to infinity, this explains why flux may be lost in our Fig. 4 (left) for negative $\alpha$ while none is lost for positive $\alpha$. Further correspondence to our Fig. 4 (left) can be found in Hudson \& Wheatland (1999)'s Fig. 2, where black points represent field lines that were "not followed all the way 

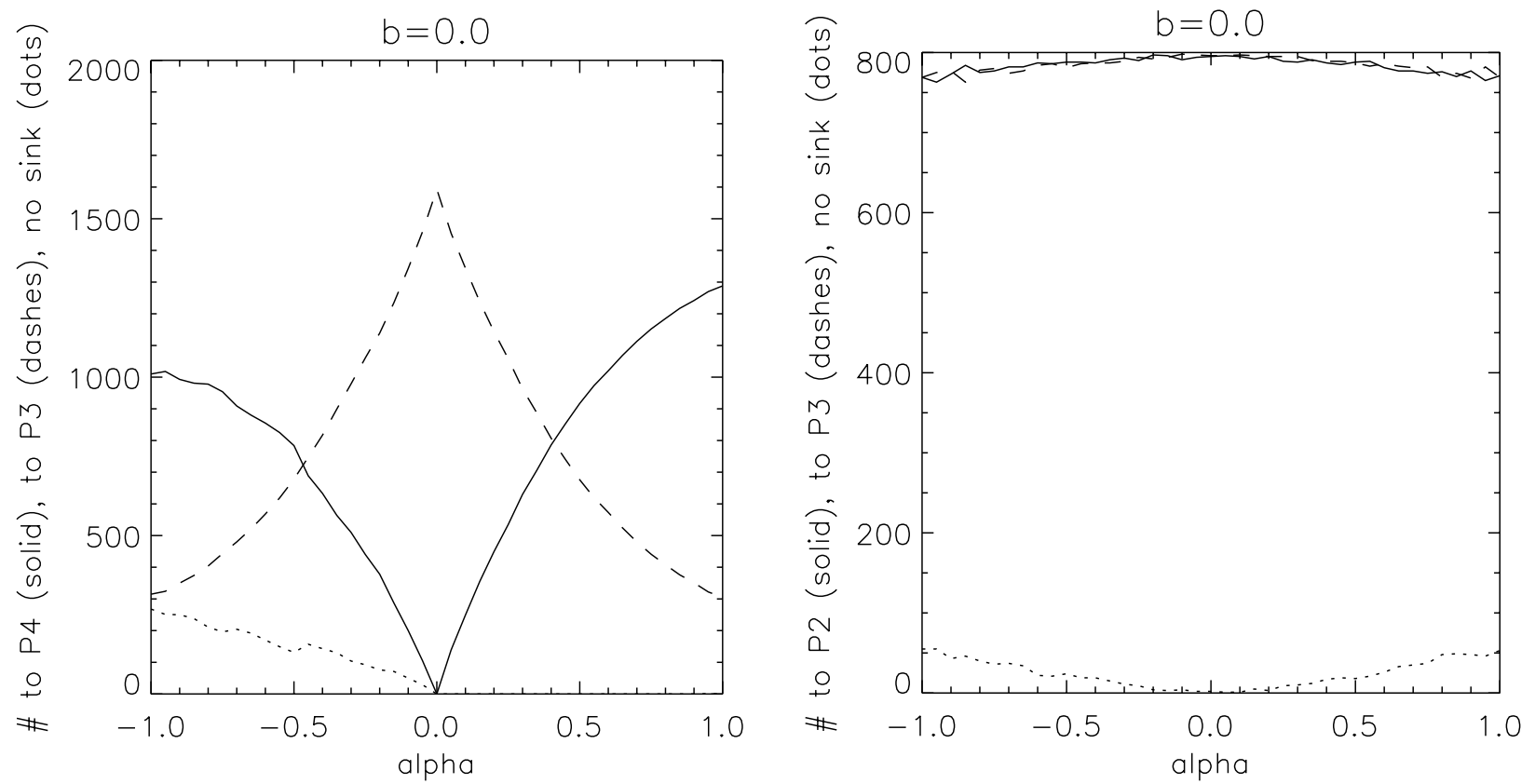

Fig. 4. Variation of field line destination with $\alpha$. In the left picture unit point magnetic flux sources are put at $(x, y, z)=P 1=(-1,1,0)$, $P 2=(1,1,0)$ and unit point flux sinks at $(x, y, z)=P 3=(-1,-1,0), P 4=(1,-1,0)$ while in the right picture unit point magnetic flux sources are put at $(x, y, z)=P 1=(-1,1,0), P 4=(1,-1,0)$ and unit point flux sinks at $(x, y, z)=P 2=(1,1,0), P 3=(-1,-1,0)$. The left picture shows graphs of flux to sink P4 (solid line), flux to sink P3 (dashed line) and flux not traced to a sink (dotted line). Meanwhile the right picture shows graphs of flux to sink P2 (solid line), flux to sink P3 (dashed line) and flux not traced to a sink (dotted line). The same-side configuration is much more prone to flux transfer between its two sinks on varying $\alpha$ than the alternate configuration.

to a charge because the tracing was halted after a certain period of time". There is a significant number of these points, and these are all to be found in the infinite connectivity region just as our missing lines are.

The only study directly comparable to our connectivity measurements in both aim and practical approach is the one by Hudson \& Wheatland (1999) in their Sect. 3.3.2. Where our results overlap with theirs, connectivity graphs for positive $\alpha$, the agreement is very good. There are regrettably no comparable results available for negative $\alpha$. Nevertheless, in the absence of proofs and comparable results, correspondence can be found between our negative- $\alpha$ results and the papers by Hudson \& Wheatland (1999) and Brown \& Priest (2000) as we have shown. In this paper we cannot account physically for this lost flux but this flux loss need not be surprising from a physical point of view. After all, when the total flux emerging from the photosphere is non-zero (the generic case), this flux must escape to infinity. There is no obvious reason why, in the zero net flux case, some flux of each sign should not escape to infinity. We speculate that this is the actual destination of much of the missing flux. Linear force-free fields are well known to have unphysical properties in infinite space, such as infinite energy in the half-space (e.g. Chiu \& Hilton 1977; Lothian \& Browning 1995). Such properties are tolerated in local models because linear force-free models are assumed invalid at large distances (Gary 1989). In some of our examples, substantial flux is lost, making a definitive interpretation difficult. However, for practical purposes, it is enough to know that this flux leaves the neighbourhood of the source distribution. In reality, such flux links to neighbouring field structures and the global solar field.

In the alternate configuration, $\alpha$ has a much smaller effect on the field line connectivity. In the potential case equal amounts of flux from $P_{1}$ reach $P_{2}$ and $P_{3}$, reflecting the symmetry of the configuration. For positive $\alpha$, the anti-clockwise spiralling of the field causes some (but not much) flux from $P_{1}$ to switch from $P_{3}$ to $P_{2}$ while a negative value for $\alpha$ brings about an opposite switch. However, because of lost flux, little can be said with confidence, except that the effect of varying $\alpha$ on this configuration is marginal.

The effect of introducing the complementary Green's function is less simple. The connectivity graphs for the same-side configuration are shown in Fig. 5, where field line destination is graphed against $b$ for two different values of $\alpha$ : 0.5 (left) and 1.0 (right). For a fixed value of $\alpha$, varying $b$ has a strong effect on the field line connectivity, but not a simple one. The effect of $b$ is greater for the larger value of $\alpha$ and the behaviour for positive $b$ is more complex than that for negative $b$. Recall that in the case $\alpha=0$ all flux goes to $P_{3}$ and none to $P_{4}$. The flux imbalance between the two sinks due to the non-zero value of $\alpha$ in favour of $P_{4}$ in the graphs of Fig. 5 is diminished by a small negative value for $b$. This effect increases with the size of $b$ until, at about $b=-1$, the flux is shared equally between the two sinks. As $b$ decreases past -1 , its effect continues to increase and the flux becomes ever more unbalanced in favour now of $P_{3}$, this effect increasing monotonically with the size of $b$. In this configuration, the complementary Green's function with a negative coefficient $b$ can be seen to be acting against 

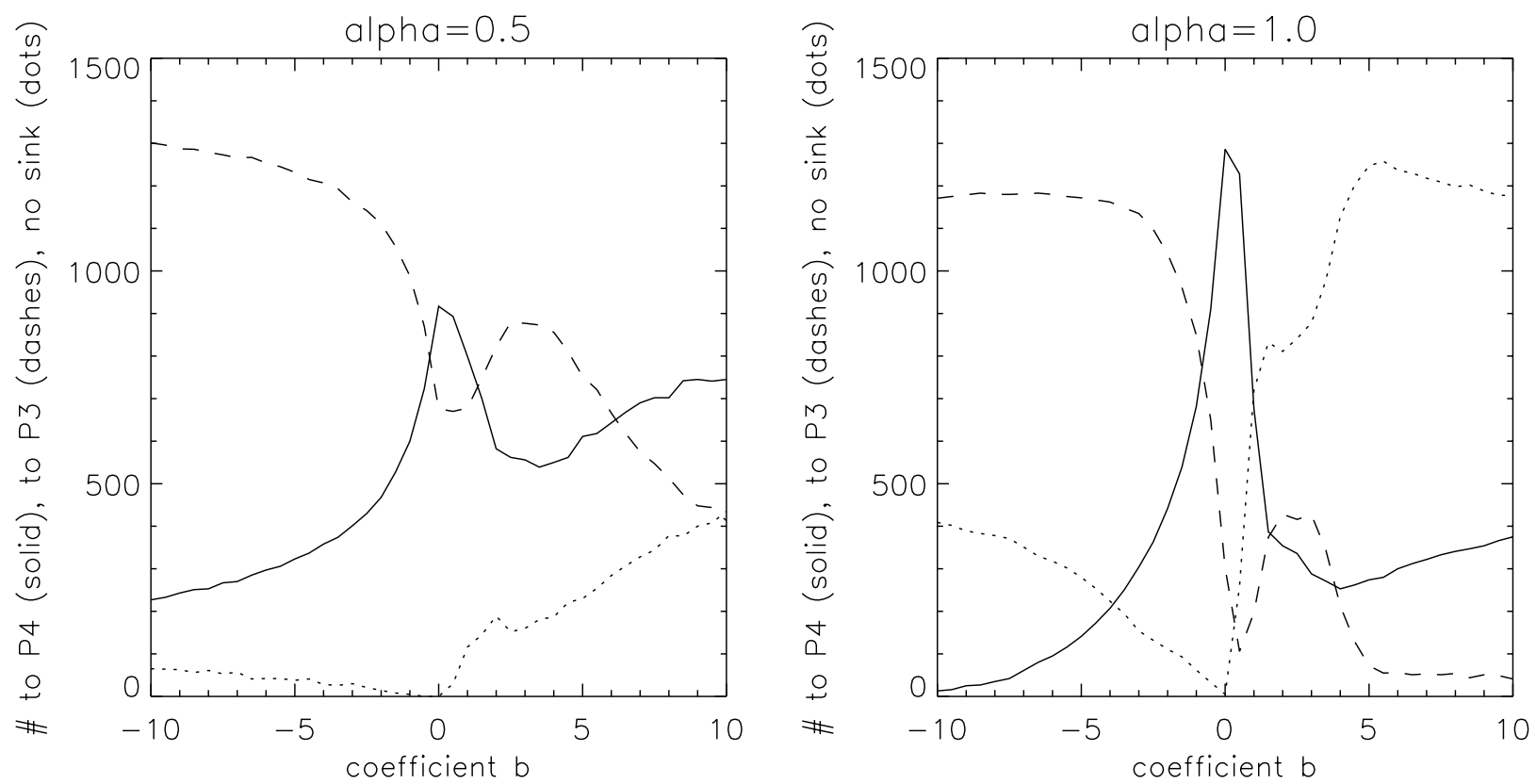

Fig. 5. Variation of field line destination with $b$. Unit point magnetic flux sources are put at $(x, y, z)=P 1=(-1,1,0), P 2=(1,1,0)$ and unit point flux sinks at $(x, y, z)=P 3=(-1,-1,0), P 4=(1,-1,0)$. The left picture shows the case $\alpha=0.5$ and the right picture shows the case $\alpha=1.0$. The two pictures show graphs of flux to sink P4 (solid line), flux to sink P3 (dashed line) and flux not traced to a sink (dotted line). The complementary Green's function has a great influence on the connectivity of this configuration according to both pictures, although for positive values of $b$ much of the flux is not traced to a sink.
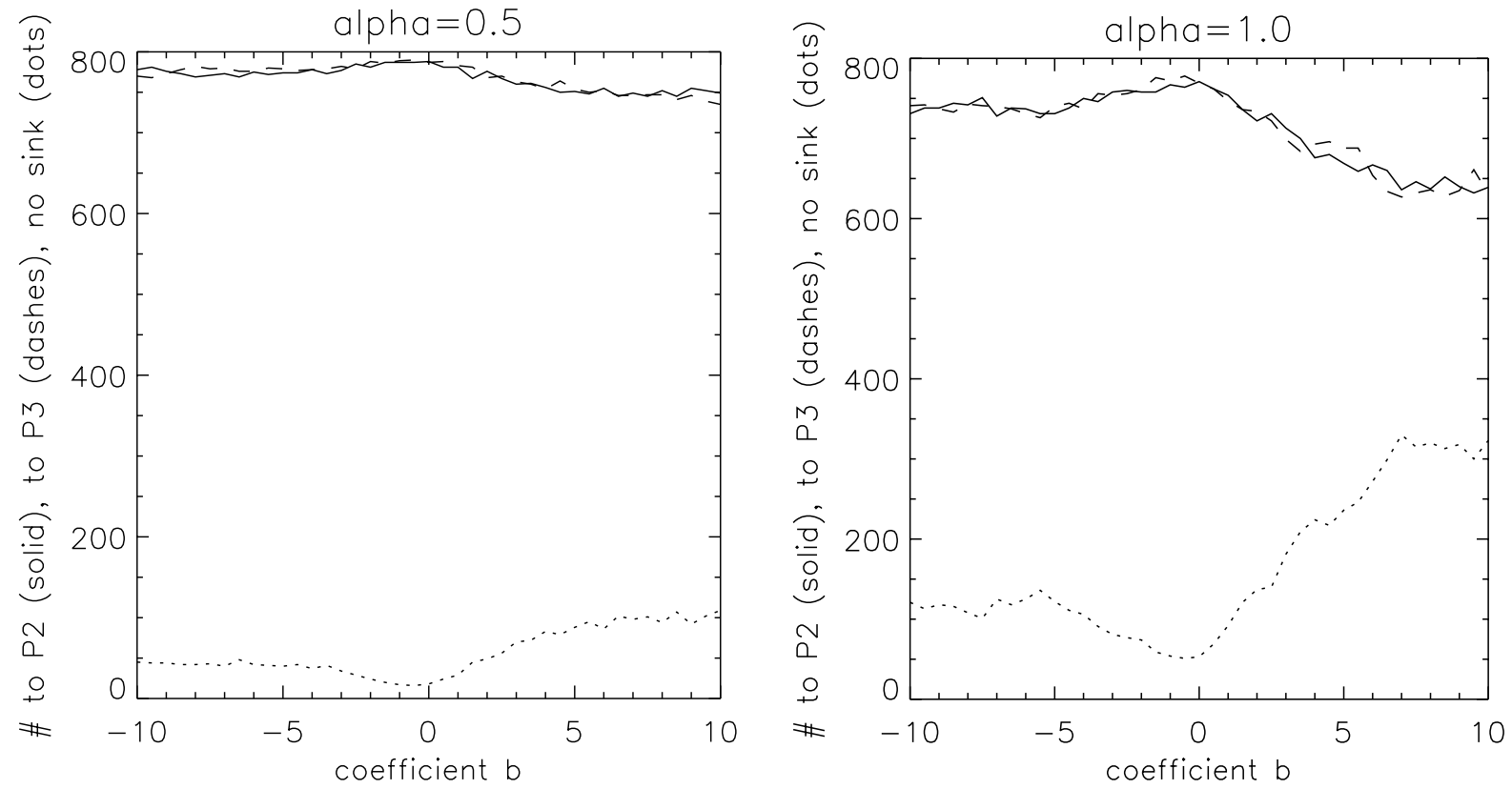

Fig. 6. Variation of field line destination with $b$. Unit point magnetic flux sources are put at $(x, y, z)=P 1=(-1,1,0), P 4=(1,-1,0)$ and unit point flux sinks at $(x, y, z)=P 2=(1,1,0), P 3=(-1,-1,0)$. The left picture shows the case $\alpha=0.5$ and the right picture shows the case $\alpha=1.0$. The two pictures show graphs of flux to sink P2 (solid line), flux to sink P3 (dashed line) and flux not traced to a sink (dotted line). For this configuration the effect of the complementary Green's function is not as great as for the same-side configuration of Fig. 5, in the same way that the effect of varying $\alpha$ is much stronger on the same-side configuration than on the alternate configuration (see Fig. 4).

the effect of the positive $\alpha$ in a simple way, redistributing flux to $P_{3}$. For small values of $\alpha$, positive values of $b$ alter the connectivity in a manner almost as simple as do negative values of $b$, tending instead to help $\alpha$ to send flux to $P_{4}$ at the expense of $P_{3}$. In this sense $b$ acts with $\alpha$ and the amount of flux to $P_{4}$ increases with the value of $b$. It can be seen from Fig. 5, however, that for larger values of $\alpha$ the situation is more complicated. In these cases, $\alpha=0.5$ and $\alpha=1.0$, there is a local maximum flux distribution to the sink at $P_{4}$ near $b=0$. Small positive values for $b$ seem to act against $\alpha$ in switching flux 
from $P_{4}$ to $P_{3}$ just like negative values of $b$, giving the graphs a locally symmetric appearance. As $b$ increases the flux to $P_{4}$ has a local minimum at about $b=3$, before steadily increasing with $b$ for $b>3$, although the details of this increase cannot be given with much confidence. Note the large amounts of lost flux in the positive half of Fig. 5 (right). This makes a definitive analysis of connectivity in this part of parameter space impossible. However, we can conclude that most of the field lines are very long in these solutions, which is, in itself, a qualitative change in field structure. For negative values of $\alpha$ connectivity graphs are similar to those of Fig. 5 except that for small values of $\alpha$ connectivity to $P_{4}$ graphed against $b$ is a monotonically decreasing function, with a local maximum appearing at $b=0$ for larger $\alpha$, and more flux is lost in this case. I.e. the graphs for negative $\alpha$ are similar to those of Fig. 5 under reflection in the line $b=0$, although the details of the connectivity for negative- $\alpha$ cases are obscured by the loss of flux.

The connectivity graphs for the alternate configuration are shown in Fig. 6, where again field line destination is graphed against $b$ for the $\alpha$ values 0.5 (left) and 1.0 (right). Here the effect of $b$ is much weaker than in Fig. 5. There is some lost flux, although not as much as in the same-side configuration. The known connectivity data do not vary much. The complementary Green's function does not influence the connectivity of this configuration much, particularly for small $\alpha$. Connectivity graphs for negative $\alpha$ values are very similar to those for positive $\alpha$ values. This, along with the small connectivity changes of this configuration on varying $\alpha$ (Fig. 4, right), suggests that the alternate configuration is much more robust against connectivity changes than the same-side configuration.

\section{Conclusions}

We have studied the effect of including a contribution from the complementary solution on some simple magnetic field structures. It was necessary to consider a restricted form for this contribution, in order to represent it by a single parameter $b$. However, using this form, we have been able to demonstrate the importance of the complementary solution. Using reasonable values for both $\alpha$ and $b$, we were able to show that they have comparable effects. The change in structure with variation of $b$ is less straightforward than the familiar response to varying $\alpha$. The customary neglect of the complementary solution has been shown to have a significant effect on the field, despite the absence of any physical justification for this neglect. However, we must remember that the constant- $\alpha$ model is itself an approximation. It could be argued that variation of $\alpha$ gives enough freedom to obtain a qualitative match to real fields and that this is sufficient for most purposes. We have measured the change in field line connectivity for two configurations involving two flux sources and two sinks. For the topologically unstable configuration studied by previous authors (Lothian \& Browning 1995; Hudson \& Wheatland 1999; Brown \& Priest 2000), variation of $b$ had a dramatic effect. Again, this effect was not as simple as that caused by increasing $\alpha$. Hence, setting $b$ equal to zero throughout constitutes a substantial assumption in previous work. Qualitatively, the potential field still changes topology (losing its separatrix) when $\alpha$ becomes non-zero. However,the detailed structure and field line connectivity depend on which constant- $\alpha$ field is chosen. The second configuration showed little change in connectivity in response to variation in either parameter. This is in agreement with the distinction between topologically stable and unstable structures emphasised by Brown \& Priest (2000).

We conclude that use of the nominal solution, as is widespread, actually selects a very special subset of the possible constant- $\alpha$ fields. This solution has previously been adopted for computational simplicity. It can now be seen that another (perhaps more important) advantage of the nominal solution is the simple way in which it responds to increasing $\alpha$. For example, estimates of $\alpha$ from EUV images, as in Avgoustidis et al. (2002), rely on such simple, predictable behaviour. There remains no strong physical reason to favour the nominal solution, but its usefulness in practice is evident.

Acknowledgements. It is a pleasure to thank Dr. Thomas Neukirch for useful discussions. GP acknowledges funding by the EU Research Training Network PLATON, contract number HPRN-CT-2000-00153.

\section{References}

Alissandrakis, C. E. 1981, A\&A, 100, 197

Avgoustidis, A., Lothian, R. M., Bromage, B. J. I., \& Walsh, R. W. 2002, ESA SP-508, 431

Amari, T., Aly, J. J., Luciani, J. F., Boulmezaoud, T. Z., \& Mikic, Z. 1997, Sol. Phys., 174, 129

Barbosa, D. D. 1977, Sol. Phys., 56, 55

Brown, D. S., \& Priest, E. R. 2000, Sol. Phys., 194, 197

Chiu, Y. T., \& Hilton, H. H. 1977, ApJ, 212, 873

Clegg, J. R., Bromage, B. J. I., \& Browning, P. K. 1999, JGR, 104, 9831

Gary, G. A. 1989, ApJS, 69, 323

Hudson, T. S., \& Wheatland, M. S. 1999, Sol. Phys., 186, 301

Longcope, D. W. 1996, Sol. Phys., 169, 91

Lothian, R. M., \& Browning, P. K. 1995, Sol. Phys., 161, 289

Petrie, G. J. D., \& Neukirch, T. 2000, A\&A, 356, 735

Petrie, G. J. D. 2000, Three-dimensional Equilibrium Solutions to the Magnetohydrodynamic Equations, Ph.D. Thesis, Univ. of St. Andrews

Schmidt, H. V. 1964 in ASS-NASA Symposium on the Physics of Solar Flares, ed. W. N. Ness (NASA SP-50), 107

Semel, M. 1988, A\&A, 198, 293

Taylor, J. B. 1974, Phys. Rev. Lett., 33, 1139

Wheatland, M. S. 1999, ApJ, 518, 948 